\title{
Intestinal perforation caused by fishbone in a child with the misdiagnosis of acute appendicitis: A case report
}

\author{
Sang Ngoc Nguyen ${ }^{1}$, Tuan Nguyen ${ }^{2}$, Lam $\mathrm{Vu}^{1}$, and Cuong Hoang ${ }^{1}$ \\ ${ }^{1}$ Hai Phong University of Medicine and Pharmacy \\ ${ }^{2}$ Haiphong Children's Hospital, Vietnam
}

May 10, 2021

\begin{abstract} examination and diagnostic imaging, are needed to make an accurate diagnosis.

Sang Ngoc Nguyen ${ }^{1 *}$, Tuan Duy Nguyen ${ }^{2}$, Lam Tung Vu ${ }^{1}$, Cuong Ngoc Bao Hoang ${ }^{1}$

*Corresponding author: Sang Ngoc Nguyen: nnsang@hpmu.edu.vn

${ }^{1}$ Haiphong University of Medicine and Pharmacy, Vietnam

${ }^{2}$ Haiphong Children's Hospital, Vietnam
\end{abstract}

Fishbone perforation, a severe complication causing damage to nearby organs, is difficult to diagnose because the patient and family cannot remember the history of fishbone ingestion exactly. A detailed history of eating, as well as a thorough physical

\section{ABSTRACT}

Fishbone perforation, a severe complication causing damage to nearby organs, is difficult to diagnose because the patient and family cannot remember the history of fishbone ingestion exactly. A detailed history of eating, as well as a thorough physical examination and diagnostic imaging, are needed to make an accurate diagnosis.

\section{Key Clinical Message}

Families should not feed their children with fish that still has its bones inside. However, fish has excellent health benefits for children. Therefore, parents need to remove all the bones from the fish before their child consumes it.

Keywords: intestinal perforation, fishbone, children

\section{INTRODUCTION}

Fishbone perforation was described by several authors in the medical literature (1-4). Perforation of the fishbone is a dangerous condition that is seldom observed. Organ damage can result from intestinal perforation, particularly peritonitis, a severe medical condition that necessitates surgery. We present a case of fishbone perforation in a 5-year-old boy who was treated at Haiphong Children's Hospital, Vietnam. 


\section{CASE PRESENTATION}

A 5-year-old boy with normal medical history was admitted to Haiphong Children's Hospital with abdominal pain around the navel at the $5^{\text {th }}$ hour of the symptom. He also had a mild fever and an inability to pass stool or gas. Physical examination: the boy was conscious, able to communicate but tired, and suffering from a fever $\left(37,5^{\circ} \mathrm{C}\right)$. His pulse rate was 100 beats per minute; his blood pressure was $90 / 60 \mathrm{mmHg}$; the respiratory rate was 30 breaths per minute. He stood at $100 \mathrm{~cm}$ tall and weighed $18 \mathrm{~kg}$. All of these numbers were in the normal range for his age. The abdominal examination noted that he has abdominal distension and obvious abdominal muscle guarding in the right iliac and umbilical region. His complete blood count: white blood cell count was $14.8 \times 10^{9} / \mathrm{l}$, neutrophils accounted for $75.8 \%$, serum CRP was $21 \mathrm{mg} / \mathrm{l}$. The abdominal ultrasound result showed that his intestines contained fluid and increase motility. The abdominal X-ray did not show abnormal images (See figure 1).

He was diagnosed with acute appendicitis and had had laparoscopic surgery. We saw little fluid sticking around intestines through the laparoscopic camera, with no pseudomembrane, no Douglas fluid, and the appendix was normal. While examining the ileum, we did not found Meckel's diverticulums but a sharp foreign object that punctured the intestine from the lumen of the intestine to the outside, about $35 \mathrm{~cm}$ from the ileum - cecum (See figure 2, 3). After that, we removed the foreign object, stitched the hole in the intestine, cleaned the abdomen, and closed incisions.

The boy made a full recovery and was discharged in a satisfactory condition following 7 days of treatment. 


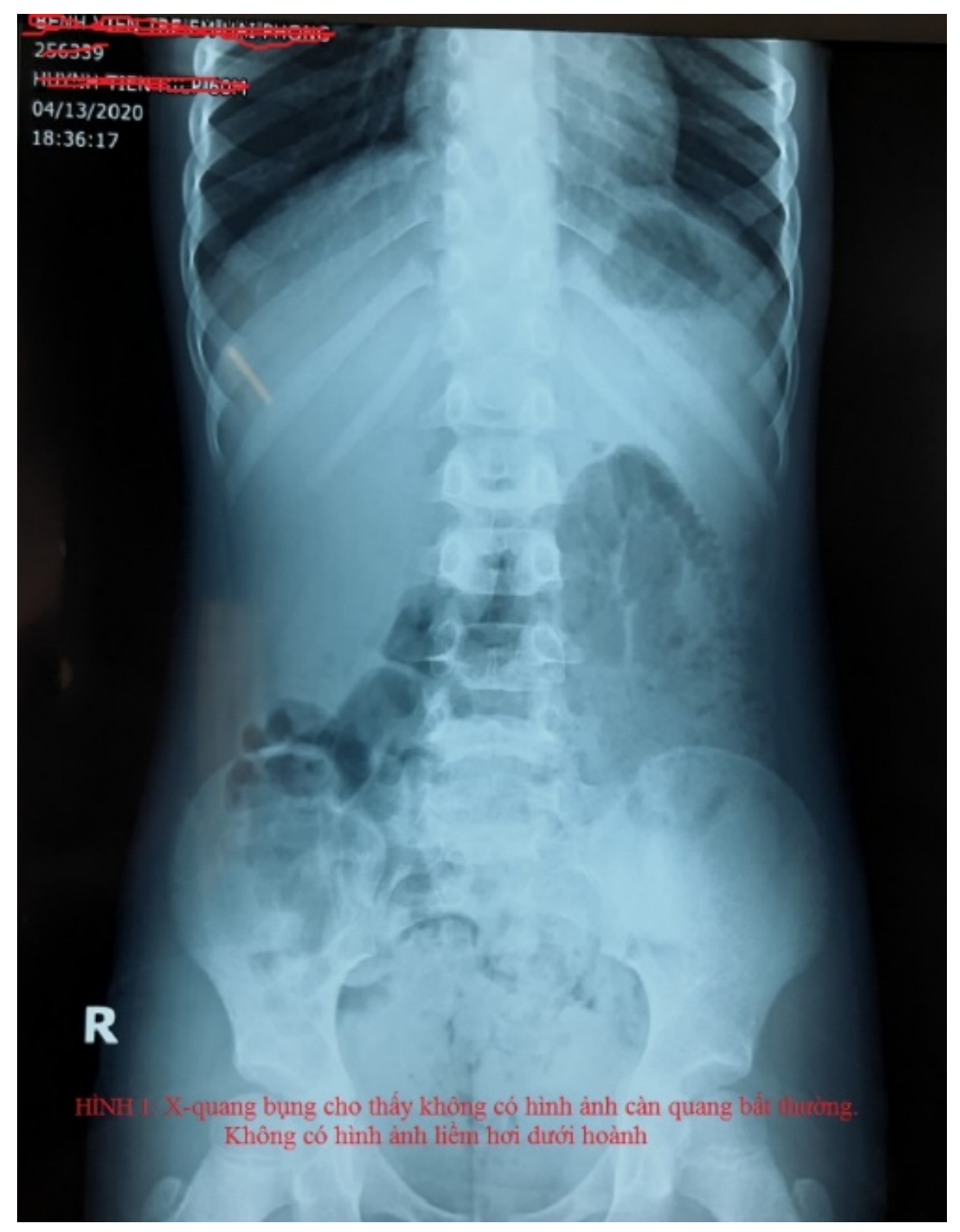

Figure 1. The patient's abdominal X-ray

\section{DISCUSSIONS}

Fishbone perforation is rarely seen, especially in children. Although most foreign objects can be excreted within a week of entering the gastrointestinal tract $(1,5), 1 \%$ of patients may still suffer from intestinal perforation if these fishbones were long or sharp. Moreover, it could be complicated to make a precise diagnosis because patients or their families cannot make sure what foreign object they ate in their meals. So, these cases are often misdiagnosed with acute appendicitis.

Depending on the hole's location, patients may have various clinical symptoms, including constipation, abdominal pain, and anal pain. A study reported that $95 \%$ of patients presented with abdominal pain, which is the most crucial symptom, $81 \%$ having a fever, and $39 \%$ having local peritonitis (2). In most cases of intestinal perforation caused by a foreign object, the foreign agent is a fishbone with a pointed shape. 
In some countries or regions that prefer eating fish, gastric perforation or other complications caused by fishbone are very common (6). While fish bones may induce intestinal perforation in any section of the intestine, it is more commonly seen in areas of physiological stenosis or intestinal transitions such as the ileum or rectosigmoid junction (7). In a study, the probability of ileal perforation was $83 \%$. (8). In another report, the perforation of the end of the ileum was $38.6 \%$, but the jejunum rate was lower, just $14.3 \%$ (5).

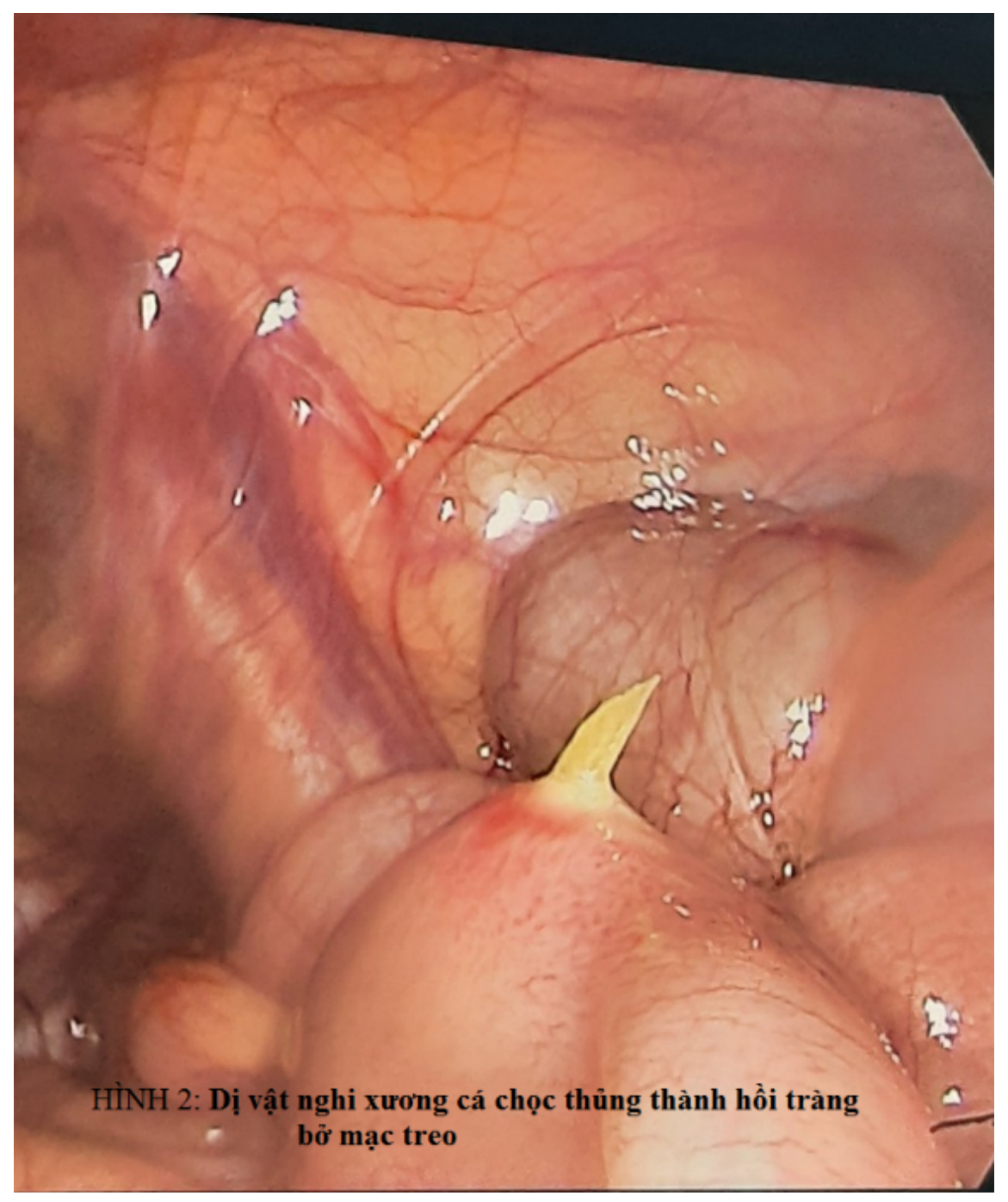

Figure 2. Fishbone punctured the intestine from the lumen of the intestine to the outside. 


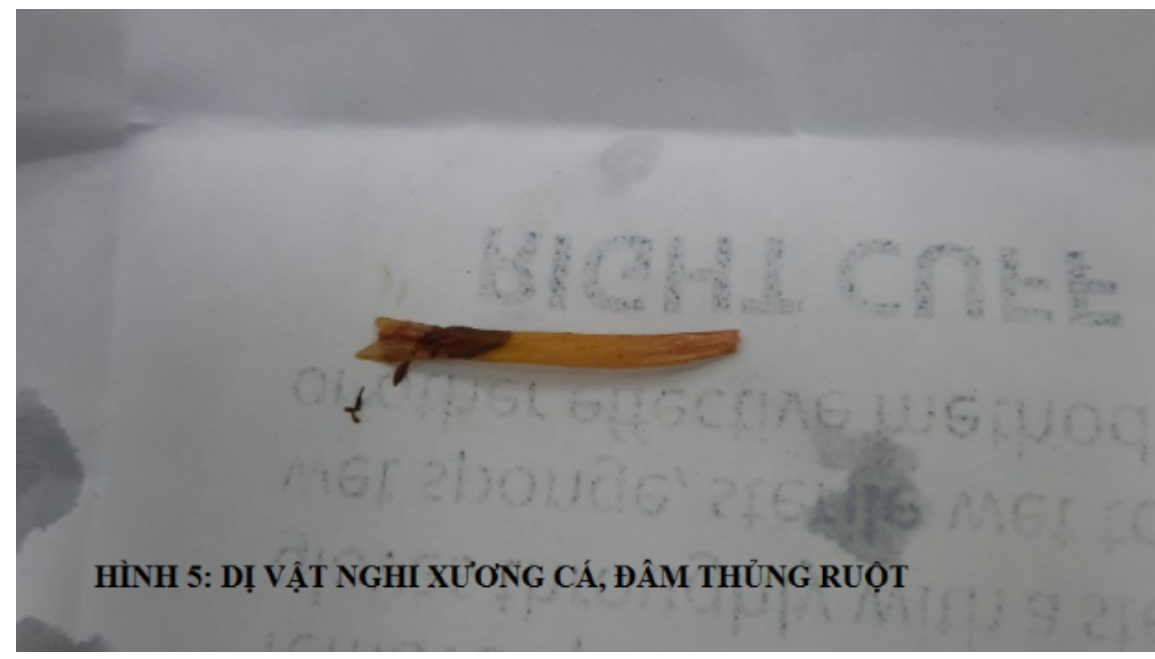

Figure 3. Full image of the fishbone.

Patients do not often think they could suffer from intestine perforation due to a fishbone. Therefore, it is hard for doctors to take a medical history of the disease. The fishbone is often only found during an imaging examination or surgical exploration (6). Imaging examination is usually unreliable in the diagnosis of these cases. High-density shadow, free gas, and abscess formation are often used to determine the presence of inflammatory changes or perforation (9). However, the fishbone gradually penetrates the intestinal wall through extrusion. The perforation site is often covered by fibrin or adjacent intestines, limiting the outflow of intestinal contents and reducing the possibility of the free gas appearance in abdominal X-ray films (10). Free intestinal gas found in X-ray films accounted for only $20 \%$ of patients (8). In another study with 358 patients suffering from fishbone perforation, X-rays were only $32 \%$ sensitive (3). The fishbone is also affected by radiation doses, inflammatory tissues, or liquids around the damage (4).

His most essential symptoms were abdominal pain, clear abdominal muscle guarding in the right iliac region with constipation, and fever in our patient. Diagnostic imaging results were not sensitive. Therefore, this patient's symptoms are somewhat close to those of a patient with acute appendicitis, perhaps leading to a misdiagnosis of abdominal pain caused by acute appendicitis.

Almost all gastrointestinal foreign bodies can be removed by gastroscopy or enteroscopy. Only $1 \%$ of cases need surgical excision. However, depending on the perforation site and clinical symptoms, treatment could be chosen through suture perforation site, bowel resection, or Hartman procedure (8). In general, surgeons prefer to remove a small bowel part to prevent perforation caused by inflammation. Laparoscopic surgery caused less damage than traditional laparoscopic surgery, so it has gradually replaced the traditional method of abdominal open exploration. Currently, laparoscopic surgery is the preferred method of choice (11). Back to our case, because there was no precise diagnosis, we chose laparoscopic surgery. During the surgery, we found a sharp fishbone punctured the intestine from the lumen of the intestine to the outside, about $35 \mathrm{~cm}$ from the ileum - cecum, and the patient's appendix was normal. We removed the fishbone, stitched the hole, cleaned the abdomen, and closed incisions.

The boy recovered very well and was discharged after 7 days of treatment with a stable condition. Nevertheless, after all of this, his family still cannot remember clearly that whether their son ate fish that day or not. All they can make sure that their family regularly ate fish for dinner. 


\section{CONCLUSION}

Identifying a case of fishbone ingestion is difficult because patients often do not remember when they ate fish, especially children. Therefore, it is critical for parents to recall their children's food and drinking history. In the case of a child with acute abdomen, doctors have to ask carefully about eating and drinking histories, if they ate fish, chicken, and so on, or not. A clinical examination together with imaging diagnosis is necessary to make the most accurate diagnosis. When performing an inflammatory appendectomy, the abdomen should also be carefully examined.

\section{RECOMMENDATIONS}

Families should not feed their children with fish that still has its bones inside. However, fish has excellent health benefits for children. Therefore, parents need to remove all the bones from the fish before their child consumes it.

\section{CONSENT}

Written informed consent was obtained from the patient and his parent for publication of these data and the accompanying images. A copy of the written consent is available for review by the Editor of this journal.

\section{CONFLICT OF INTERESTS}

The authors declare that they have no competing interests.

\section{AUTHORS' CONTRIBUTIONS}

SNN, TDN, LTV, and CNBH participated in the study design, protocol development, and performance, data analysis, interpretation of data, writing the manuscript, carrying out the clinical data collection and data analysis, and observing the patient during the treatment. TDN and CNBH performed laparoscopic surgery. All authors read and approved the final manuscript.

\section{ACKNOWLEDGMENTS}

The authors would like to thank the patient and his family in this study for their cooperation.

\section{REFERENCES}

1. Song J, Yang W, Zhu Y, Fang Y, Qiu J, Qiu J, et al. Ingested a fish bone-induced ileal perforation: A case report. Medicine (Baltimore). 2020;99(15):e19508.

2. Emir S, Ozkan Z, Altinsoy HB, Yazar FM, Sozen S, Bali I. Ingested bone fragment in the bowel: Two cases and a review of the literature. World J Clin Cases. 2013;1(7):212-6.

3. Ngan JH, Fok PJ, Lai EC, Branicki FJ, Wong J. A prospective study on fish bone ingestion. Experience of 358 patients. Ann Surg. 1990;211(4):459-62. 
4. Coulier B, Tancredi MH, Ramboux A. Spiral CT and multidetector-row CT diagnosis of perforation of the small intestine caused by ingested foreign bodies. Eur Radiol. 2004;14(10):1918-25.

5. Rodriguez-Hermosa JI, Codina-Cazador A, Sirvent JM, Martin A, Girones J, Garsot E. Surgically treated perforations of the gastrointestinal tract caused by ingested foreign bodies. Colorectal Dis. 2008;10(7):701-7.

6. Joglekar S, Rajput I, Kamat S, Downey S. Sigmoid perforation caused by an ingested chicken bone presenting as right iliac fossa pain mimicking appendicitis: a case report. J Med Case Rep. 2009;3:7385.

7. Glasson R, Haghighi KS, Richardson G. Chicken bone perforation of a sigmoid diverticulum. ANZ J Surg. 2002;72(6):448-9.

8. Singh RP, Gardner JA. Perforation of the sigmoid colon by swallowed chicken bone: case reports and review of literature. Int Surg. 1981;66(2):181-3.

9. Akhtar S, McElvanna N, Gardiner KR, Irwin ST. Bowel perforation caused by swallowed chicken bones-a case series. Ulster Med J. 2007;76(1):37-8.

10. Pinero Madrona A, Fernandez Hernandez JA, Carrasco Prats M, Riquelme Riquelme J, Parrila Paricio P. Intestinal perforation by foreign bodies. Eur J Surg. 2000;166(4):307-9.

11. Chin EH, Hazzan D, Herron DM, Salky B. Laparoscopic retrieval of intraabdominal foreign bodies. Surg Endosc. 2007;21(8):1457. 